\title{
Geometric Photon-Drag Effect and Nonlinear Shift Current in Centrosymmetric Crystals
}

\author{
Li-kun Shi ${ }^{1,2, *}$ Dong Zhang, ${ }^{3,4,5}$ Kai Chang, ${ }^{3,4,5}$ and Justin C. W. Song ${ }^{1,6, \dagger}$ \\ ${ }^{1}$ Division of Physics and Applied Physics, Nanyang Technological University, Singapore 637371, Republic of Singapore \\ ${ }^{2}$ Max Planck Institute for the Physics of Complex Systems, 01187 Dresden, Germany \\ ${ }^{3}$ SKLSM, Institute of Semiconductors, Chinese Academy of Sciences, P.O. Box 912, Beijing 100083, China \\ ${ }^{4}$ CAS Center for Excellence in Topological Quantum Computation, University of Chinese Academy of Sciences, Beijing 100190, China \\ ${ }^{5}$ Beijing Academy of Quantum Information Sciences, Beijing 100193, China \\ ${ }^{6}$ Institute of High Performance Computing, Agency for Science, Technology, \& Research, Singapore 138632, Republic of Singapore
}

(Received 2 July 2020; accepted 22 March 2021; published 10 May 2021)

\begin{abstract}
The nonlinear shift current, also known as the bulk photovoltaic current generated by linearly polarized light, has long been known to be absent in crystals with inversion symmetry. Here we argue that a nonzero shift current in centrosymmetric crystals can be activated by a photon-drag effect. Photon-drag shift current proceeds from a "shift current dipole" (a geometric quantity characterizing interband transitions) and manifests a purely transverse response in centrosymmetric crystals. This transverse nature proceeds directly from the shift-vector's pseudovector nature under mirror operation and underscores its intrinsic geometric origin. Photon-drag shift current can be greatly enhanced by coupling to polaritons and provides a new and sensitive tool to interrogate the subtle interband coherences of materials with inversion symmetry previously thought to be inaccessible via photocurrent probes.
\end{abstract}

DOI: 10.1103/PhysRevLett.126.197402

The bulk photovoltaic effect produces a photocurrent in a single-phase homogeneous material [1-6] that persists even in the absence of conventional $p-n$ junctions. This renders an entire bulk material active in photocurrent generation. A prominent example of bulk photovoltaic effect is the nonlinear shift current [7-18] wherein geometric phases sustained by electronic states $[19,20]$ enable a photoinduced current in the bulk. While such geometric phases can be found in a large variety of materials, since photocurrent is a vector, broken (intrinsic) symmetries are required in fixing the direction of shift current in a uniform bulk. As a result, shift currents are typically thought to vanish in centrosymmetric materials [4-7], even in those possessing nontrivial geometric phases.

Here we show that nonzero shift currents can be revived in centrosymmetric crystals. In particular, we find that nonvertical transitions [Fig. 1(b)] [21], readily enabled by photon or polariton-drag processes, produce finite shift currents even when crystal inversion symmetry remains unbroken. While requiring a finite momentum transfer, such photon-drag shift currents are intrinsic with a magnitude controlled by a "shift-current dipole" that captures the interband geometry present in a material; this closely

Published by the American Physical Society under the terms of the Creative Commons Attribution 4.0 International license. Further distribution of this work must maintain attribution to the author(s) and the published article's title, journal citation, and DOI. Open access publication funded by the Max Planck Society. parallels the Berry curvature dipole [22] describing intraband geometry.

Surprisingly, photon-drag shift currents are transverse to the momentum transfer in isotropic crystals with a longitudinal incident polarization. As we explain below, this transverse nature arises from an intrinsic helical winding of electronic states found in many (centrosymmetric) systems (e.g., $\mathrm{HgTe}$ quantum wells, monolayer $1 \mathrm{~T}^{\prime}-\mathrm{MX}_{2}$ systems) and vividly displays its geometric origin. This intrinsic behavior sharply contrasts with conventional photon-drag that is parallel or antiparallel to the momentum transfer for a longitudinal polarization [23-26]. We note that transverse currents are more typically found when using nonzero photon angular momentum or in structured media [27-33].

We expect that photon-drag shift current can be found in a wide variety of centrosymmetric materials and can serve as a sensitive diagnostic of their interband geometry using readily available photocurrent spectroscopy-previously thought impossible [4-7]. This opens up a vast set of centrosymmetric materials to realize geometrical photocurrents.

Shift current and photon-drag.-First analyzed by von Baltz and Kraut [8], the shift current (density) arises from real-space displacements of an electron accrued during a photoinduced transition from an initial to a final state:

$$
\mathbf{j}^{s}=e \sum_{i \rightarrow f} W_{i \rightarrow f} \mathbf{r}_{i \rightarrow f},
$$

where $i$ and $f$ denote initial and final electronic states in momentum space, $W_{i \rightarrow f}$ is the photoinduced transition 
(a) vertical transitions

(b) nonvertical transitions

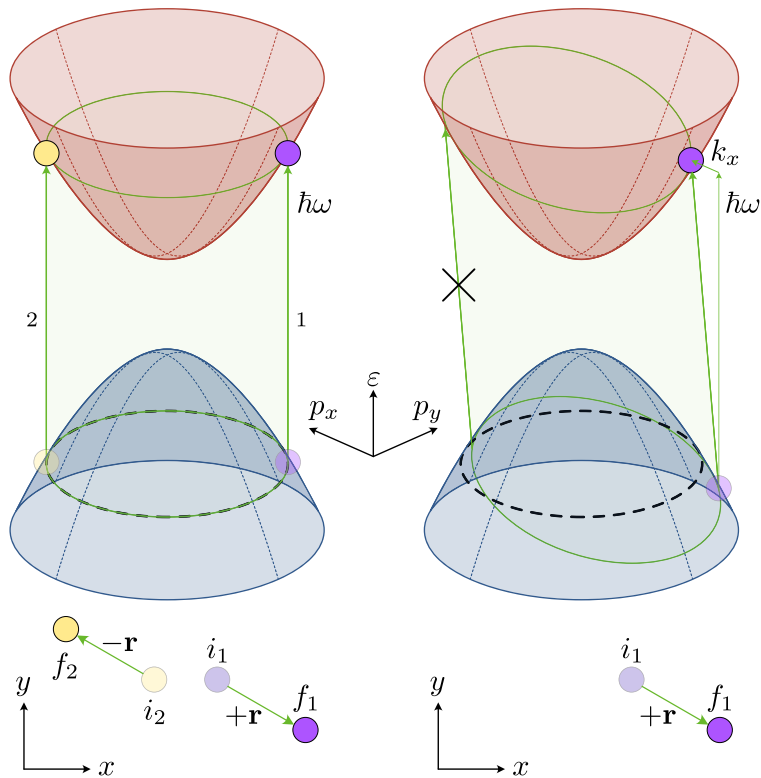

FIG. 1. Comparison between (a) vertical transitions and (b) nonvertical transitions (e.g., from photon drag) between two bands. Dashed black contours denotes the Fermi surface (FS) in the valence band (blue pockets). Green contours are energy-momentum-conserving contours (ECs) for (a) vertical transitions $\omega_{c, \mathbf{p}}-\omega_{v, \mathbf{p}}-\omega=0$ and (b) nonvertical transitions $\omega_{c, \mathbf{p}+\mathbf{k} / 2}-\omega_{v, \mathbf{p}-\mathbf{k} / 2}-\omega=0$. For nonvertical transitions, only part of the EC that lies below the FS is optically allowed (right green arrow). Top: arrows from light to dark circles in upper panels represent transitions of electrons in energy-momentum space; in the lower panels, they illustrate real space displacements. Yellow and purple circles denote electron Bloch states with opposite wave vectors.

(absorption) rate, and $\mathbf{r}_{i \rightarrow f}$ is the real-space displacement acquired during the transition.

For a vertical optical transition between a valence $(v)$ and conduction $(c)$ band, an electron's initial and final state has the same momentum, p [see Fig. 1(a)]. As a result, $W_{i \rightarrow f}=W(\mathbf{p}, v \rightarrow c)$, with $\mathbf{r}_{i \rightarrow f}$ described by the shift vector $[8,9,17,34,35]$

$$
\mathbf{r}^{(0)}(\mathbf{p})=\mathbf{A}_{c}(\mathbf{p})-\mathbf{A}_{v}(\mathbf{p})-\nabla_{\mathbf{p}} \arg \left[\nu^{(0)}(\mathbf{p})\right]
$$

where $\mathbf{A}_{c, v}(\mathbf{p})=\left\langle u_{c, v}(\mathbf{p}) \mid i \nabla_{\mathbf{p}} u_{c, v}(\mathbf{p})\right\rangle$ is the Berry connection for $c, v$ bands, and $\nu^{(0)}(\mathbf{p})=\left\langle u_{\mathrm{c}}(\mathbf{p})|\mathbf{e} \cdot \hat{\mathbf{\nu}}| u_{\mathrm{v}}(\mathbf{p})\right\rangle$, where $\mathbf{e}$ is the polarization of light, $\hat{\mathbf{v}}=\partial H(\mathbf{p}) / \hbar \partial \mathbf{p}$, with $H(\mathbf{p})$ the Bloch Hamiltonian.

For crystals with inversion symmetry (IS), the shift vector $\mathbf{r}^{(0)}(\mathbf{p})=-\mathbf{r}^{(0)}(-\mathbf{p})$ is odd in momentum space (see, e.g., below and the Supplemental Material [36]). In contrast, the transition rate $W(\mathbf{p}, v \rightarrow c)$ is even under inversion. As a result the shift current density Eq. (1) vanishes in centrosymmetric crystals [4-7].
As we now argue, this constraint can be circumvented even in centrosymmetric crystals by considering nonvertical transitions, shown in Fig. 1(b). Such nonvertical transitions readily manifest from photon drag (or polariton drag, see below), which include momentum transfer from photons to electrons: the initial and final states read as $\left|u_{v}(\mathbf{p}-\mathbf{k} / 2)\right\rangle$ and $\left|u_{c}(\mathbf{p}+\mathbf{k} / 2)\right\rangle$ with $\hbar \mathbf{k}$ the momentum transferred from the photon to the electron [21]. Accounting for these transitions in nonlinear response theory [8], see [36] for full details, we obtain a photondrag shift current density as

$\mathbf{j}^{s}(\mathbf{k})=C \int_{\mathbf{p}} \rho(\mathbf{p}, \mathbf{k}) \mathbf{R}(\mathbf{p}, \mathbf{k}), \quad \mathbf{R}(\mathbf{p}, \mathbf{k}) \equiv|\nu(\mathbf{p}, \mathbf{k})|^{2} \mathbf{r}(\mathbf{p}, \mathbf{k})$,

where $C=e(\pi / 2)(e E / \hbar \omega)^{2}$ contains the electric field strength $E$ and light frequency $\omega$, the $d$-dimensional integral is written as $\int_{\mathbf{p}} \equiv \int \mathrm{d}^{d} p_{i} /(2 \pi)^{d}$, and $\rho(\mathbf{p}, \mathbf{k})=$ $\left[f\left(\epsilon_{\mathrm{v}, \mathbf{p}-\mathbf{k} / 2}\right)-f\left(\epsilon_{\mathrm{c}, \mathbf{p}+\mathbf{k} / 2}\right)\right] \delta\left(\omega_{\mathrm{c}, \mathbf{p}+\mathbf{k} / 2}-\omega_{\mathrm{v}, \mathbf{p}-\mathbf{k} / 2}-\omega\right)$ defines a tilted, optically allowed energy-momentumconserving contour (EC) in momentum space [see Fig. 1(b)]. The velocity matrix element is $\nu(\mathbf{p}, \mathbf{k})=$ $\left\langle u_{c}(\mathbf{p}+\mathbf{k} / 2)|\hat{\nu} \cdot \mathbf{e}| u_{v}(\mathbf{p}-\mathbf{k} / 2)\right\rangle$. Crucially, real-space displacements $\mathbf{r}_{i \rightarrow f}$ for nonvertical transitions in Fig. 1(b) are

$\mathbf{r}(\mathbf{p}, \mathbf{k})=\mathbf{A}_{c}(\mathbf{p}+\mathbf{k} / 2)-\mathbf{A}_{v}(\mathbf{p}-\mathbf{k} / 2)-\nabla_{\mathbf{p}} \arg [\nu(\mathbf{p}, \mathbf{k})]$.

When $\mathbf{k}=0, \mathbf{j}^{\mathbf{s}}(0)$ in Eq. (3) reduces to the conventional shift current for vertical transitions without photon drag. Indeed, $\mathbf{r}(\mathbf{p}, \mathbf{k}=0)=\mathbf{r}^{(0)}(\mathbf{p})$ in Eq. (2). While we have focused on a two-band system in Eq. (3), the same expression can be readily applied to multiple bands by accounting for all pairs of transitions, see the full multiband formula in [36].

We note that Eq. (3) can also be obtained by applying Fermi's golden rule onto Eq. (1) [17] for the nonvertical transitions in Fig. 1(b), see [36]. This provides a simple physical and intuitive picture of the effect: due to the finite photon momentum $\mathbf{k}$, nonvertical transitions in Fig. 1(b) do not respect the crystal's inversion symmetry. Indeed, we find $\rho(\mathbf{p}, \mathbf{k}) \mathbf{r}(\mathbf{p}, \mathbf{k}) \neq-\rho(-\mathbf{p}, \mathbf{k}) \mathbf{r}(-\mathbf{p}, \mathbf{k})$ even in a centrosymmetric material and can thus produce a nonvanishing photon-drag shift current.

Shift current dipole.-To track the direction of $\mathbf{j}^{s}(\mathbf{k})$ and its dependence on $\mathbf{k}$, we expand it at small $\mathbf{k}$, representing it as a product of $\mathbf{k}$ with a "shift current dipole," $\mathbf{D}$, via

$$
j_{b}^{s}(\mathbf{k})=k_{a} D_{a b}+O\left(k^{2}\right)
$$

where $a, b=\{x, y, z\}$, and repeated indices are implicitly summed over. In a $2 \mathrm{D}$ system, $D_{a b}$ in Eq. (5) has the dimensions of current. The shift current dipole is 


$$
D_{a b}=C \int_{\mathbf{p}}\left[d_{a}^{\rho}(\mathbf{p}) R_{b}^{(0)}(\mathbf{p})+d_{a b}^{R}(\mathbf{p}) \rho^{(0)}(\mathbf{p})\right],
$$

where $R_{b}^{(0)}(\mathbf{p})$ and $\rho^{(0)}(\mathbf{p})$ are obtained at $\mathbf{k}=0$ similar to $\mathbf{r}^{(0)}(\mathbf{p}), d_{a b}^{R}(\mathbf{p})=\left[\partial R_{b}(\mathbf{p}, \mathbf{k}) / \partial k_{a}\right]_{\mathbf{k}=0}$, and

$d_{a}^{\rho}(\mathbf{p})=\left.\frac{\partial \rho(\mathbf{p}, \mathbf{k})}{\partial k_{a}}\right|_{\mathbf{k}=0}=-\frac{1}{2} \frac{\partial\left(f_{v, \mathbf{p}}+f_{c, \mathbf{p}}\right)}{\partial p_{a}} \delta\left(\omega_{\mathbf{p}}^{c v}-\omega\right)$,

with $f_{c(v), \mathbf{p}}=\left[1+e^{\left(\epsilon_{c(v) \cdot \mathbf{p}}-\mu\right) / k_{B} T}\right]^{-1}$ the Fermi function and $\omega_{\mathbf{p}}^{c v}=\omega_{c, \mathbf{p}}-\omega_{\mathrm{v}, \mathbf{p}}$; the second equality in Eq. (7) was obtained for symmetric $c, v$ bands. As we will see below, the shift current dipole is nonzero even in centrosymmetric crystals and characterizes the behavior of the photon-drag shift current.

Transverse shift current and shift current dipole.-From Eqs. (4) and (6), the shift current dipole depends on both the intrinsic properties of the crystal and the light polarization. For a generic centrosymmetric crystal without any additional crystalline symmetry, both $D_{x x}$ and $D_{x y}$ can be nonzero. However, as we now show, crystalline symmetry can severely constrain the form of the shift current dipole $D_{a b}$.

We proceed by noting that for centrosymmetric crystals with time reversal symmetry (TRS) and mirror symmetry (MS) $\left(\mathcal{M}_{y}: y \rightarrow-y\right)$, the spin-resolved generalized shift vector $[\mathbf{r}(\mathbf{p}, \mathbf{k})]^{\sigma}$ satisfy symmetry constrained relations; here $\sigma=\uparrow, \downarrow$. These symmetry constraints can be readily obtained by directly analyzing how Eq. (4) (or, equivalently, the Wilson-loop associated with the interband transitions) transforms under time-reversal and mirror operations, see details in [36]. Below we will focus on incident light with linear polarization (captured by $\hat{\nu} \cdot \mathbf{e}=\nu_{x}$ ) and the photon wave vector $\mathbf{k}=k_{x} \hat{\mathbf{x}}$ both being parallel to the mirror plane (fixed along the $x$ axis); in this case, the light electric field does not break overall MS. For a discussion of other polarizations, see [36]. For e along the mirror plane, we find

$$
\begin{aligned}
& {[\mathbf{R}(\mathbf{p}, \mathbf{k})]_{x}^{\sigma}=-\left[\mathbf{R}\left(\mathcal{M}_{y} \mathbf{p}, \mathcal{M}_{y} \mathbf{k}\right)\right]_{x}^{\sigma},} \\
& {[\mathbf{R}(\mathbf{p}, \mathbf{k})]_{y}^{\sigma}=\left[\mathbf{R}\left(\mathcal{M}_{y} \mathbf{p}, \mathcal{M}_{y} \mathbf{k}\right)\right]_{y}^{\sigma},}
\end{aligned}
$$

where $[\cdots]_{x, y}$ denote the $x, y$ components, and we have noted $|\nu(\mathbf{p}, \mathbf{k})|^{2}=\left|\nu\left(\mathcal{M}_{y} \mathbf{p}, \mathcal{M}_{y} \mathbf{k}\right)\right|^{2}$. In obtaining Eq. (8) we have repeatedly applied MS, TRS, and IS. Interestingly, Eq. (8) shows that $[\mathbf{R}(\mathbf{p}, \mathbf{k})]^{\sigma}$ behaves as a pseudovector with respect to the mirror plane. In contrast, $[\rho(\mathbf{p}, \mathbf{k})]^{\sigma}$ transforms as

$$
[\rho(\mathbf{p}, \mathbf{k})]^{\sigma}=\left[\rho\left(\mathcal{M}_{y} \mathbf{p}, \mathcal{M}_{y} \mathbf{k}\right)\right]^{\sigma},
$$

where we have similarly repeatedly applied MS, TRS, and IS.

Recalling that $\mathbf{k}=k_{x} \hat{\mathbf{x}}$ so that $\mathcal{M}_{y} \mathbf{k}=\mathbf{k}$, the transformation properties in Eqs. (8) and (9) can be directly applied to Eq. (6) enforcing a vanishing $D_{x x}^{\sigma}=0$, but allowing a finite $D_{x y}^{\sigma} \neq 0$. This can be readily obtained by analyzing the eveness and oddness of the integrand of Eq. (6) as $\mathbf{p} \rightarrow \mathcal{M}_{y} \mathbf{p}$. As a result, the shift current dipole is purely transverse. Applying the transverse $D_{x y}^{\sigma}$ onto Eq. (5) we obtain a photon-drag shift current density that is transverse to the photon wave vector $\mathbf{k}$ (when $\mathbf{k}$, together with the light polarization, is aligned with the mirror axis). We note that when the linear polarization is not directed along a mirror plane (overall mirror symmetry is broken), all components of $D_{a b}^{\sigma}$ are generically allowed.

While we have focused on the shift current dipole and the small $k$ behavior in Eq. (5), the transformation properties of Eqs. (8) and (9) are general and can also be applied to the full $[\mathbf{R}(\mathbf{p}, \mathbf{k})]^{\sigma}$ and $[\rho(\mathbf{p}, \mathbf{k})]^{\sigma}$ in Eq. (3). In the same fashion as above, we obtain a transverse photon-drag shift current for linear polarization along an in-plane mirror axis valid even for large $|\mathbf{k}|$. We emphasize both analyses [from shift current dipole or Eq. (3)] demonstrate that a transverse photon drag shift current arises due the combined action of IS, MS, and TRS. This transverse behavior contrasts with that of the standard shift current (in an IS breaking system) that flows along a mirror plane when normal incident light polarisation is parallel or perpendicular to the mirror plane [37].

Polariton enhanced photon-drag.-In most cases, the wavelength (wave vector $\mathbf{k}$ ) of light is much larger (smaller) than typical electron wavelengths (wave vectors). As a result, the photon-drag shift current [see Eq. (5)] can be small. However, as we show below, sizeable $j_{b}^{s}(\mathbf{k})$ can be achieved when coupling with polaritons [21,38,39] amplifying the wave vector $\mathbf{k}$ at the same frequency [21]. Graphene plasmons (GP) are exceptionally tailored to achieve this task because (1) its wavelength can be masssively compressed to 50 to $100 \mathrm{~nm}$ within a large frequency window [38], (2) GP can possess a large quality factor (as large as 130 [39]) and can propagate through and cover a large sample, (3) GP generates enhanced ac electric fields (of order $\sim 1-10$ times [40]) that can extend out to its surrounding environment.

As such, a layered van der Waals stacked structure (see Fig. 2) [21], can be readily employed to plasmonically enhance the photon-drag shift current in a target 2D material. By stacking a $2 \mathrm{D}$ or thin-film target crystal on top of a graphene layer with a thin insulating layer (e.g., hexagonal boron nitride that can be as thin as several $\mathrm{nm}$ ) in between, then exciting a propagating GP in the graphene, the longitudinal ac electric field generated by the GP [41,42] (whose linear polarization aligns with its large wave vector $\mathbf{k}$ ) can trigger nonvertical transitions [21] in the target layer. Taking into account the values of 


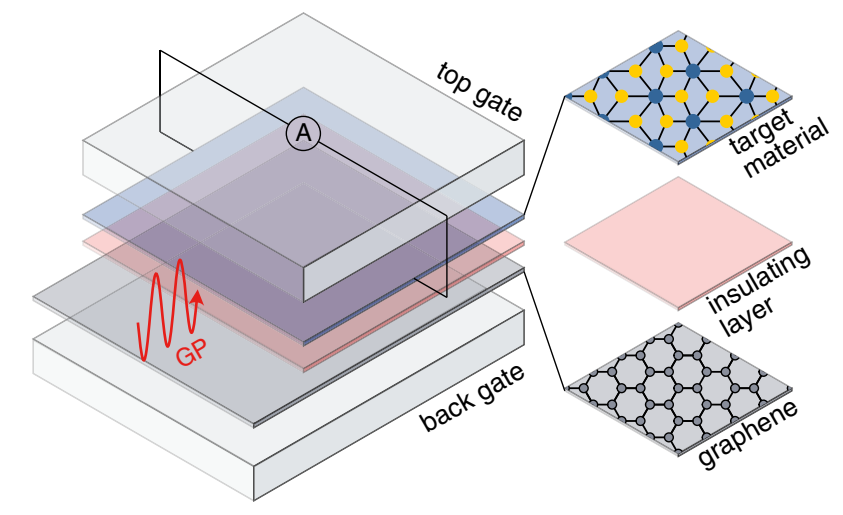

FIG. 2. Schematic of graphene plasmon enhanced photon-drag effect. A centrosymmetric 2D or thin-film target material (blue layer) is stacked on top of a graphene monolayer (thin gray layer), with an insulating layer (purple layer) in between. A propagating graphene plasmon (GP, red curve) can induce nonvertical optical transitions and generates a shift current in the adjacent target material (that can be perpendicular to the GP propagation direction, i.e., a transverse photon-drag shift current). Fermi surface of the target crystal is tunable with top and bottom gates (thick gray layers).

compression and field enhancements achievable in (1) and (3) above, we estimate order of magnitude polariton enhancements between $\sim 50-1000$ can be achieved as compared with pure photon drag.

Spin and charge transverse photon-drag shift current.We now turn to exemplify the photon-drag shift current in a minimal low-energy model of a $2 \mathrm{D}$ centrosymmetric crystal, the Bernevig-Hughes-Zhang (BHZ) model [43]

$$
H_{0}=m_{\mathbf{p}} s_{0} \tau_{z}+v_{x} p_{x} s_{z} \tau_{x}-v_{y} p_{y} s_{0} \tau_{y}
$$

describing two spin-degenerate bands with TRS, where $s_{x, y, z}$ and $\tau_{x, y, z}$ denote spin and orbital degrees of freedom, respectively; $s_{0}$ and $\tau_{0}$ are $2 \times 2$ identity matrices, $m_{\mathbf{p}}=m_{0}-\left(c_{x} p_{x}^{2}+c_{y} p_{y}^{2}\right)$. The BHZ model can characterize the low-energy electronic and optical behavior of a wide variety of systems. For example, when $c_{x}=c_{y}, v_{x}=v_{y}$ it captures an isotropic and centrosymmetric electronic system (e.g., HgTe quantum wells [43]) and when $c_{x} \neq c_{y}$ or $v_{x} \neq v_{y}$ it describes a centrosymmetric system with a mirror plane [44-46]. To clearly exhibit the pseudovector nature of $\left[\mathbf{R}^{(0)}(\mathbf{p})\right]^{\sigma}$, we concentrate on the latter with a mirror plane along the $x$ axis.

We first focus on the spin-up branch of Eq. (10) and calculate its shift current dipole, see parameter values for Eq. (10) in caption. Other parameters values can also be used with no qualitative change to our results. We note that $H_{0}^{\uparrow}$ possesses charge conjugation symmetry yielding $d_{a b}^{R, \uparrow}(\mathbf{p})=0$, see [36]. At zero temperature $T=0$ and assuming the chemical potential is in the valence band, the shift current dipole for the spin-up branch can be written as

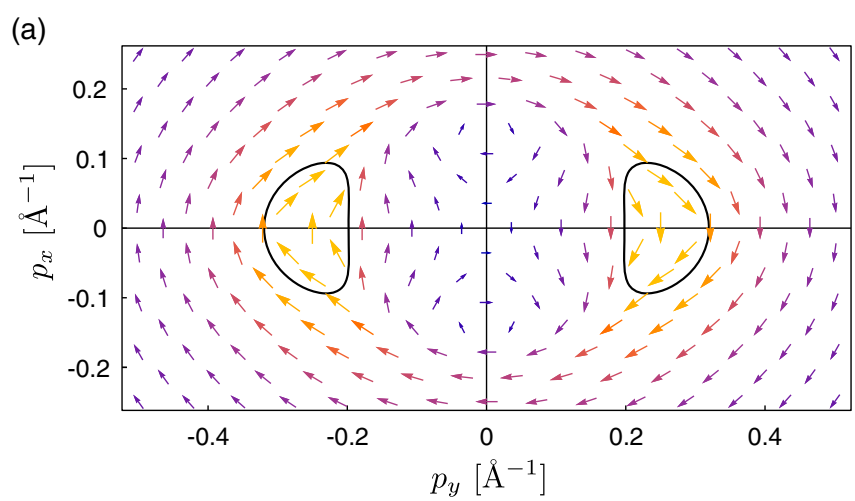

(b)
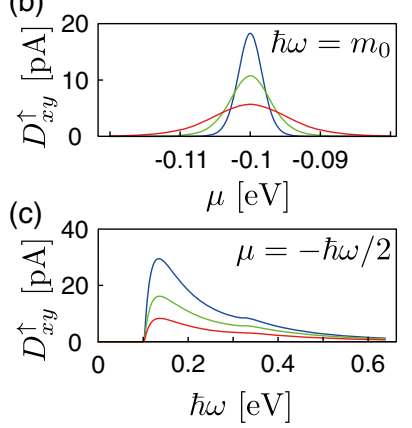

(d)

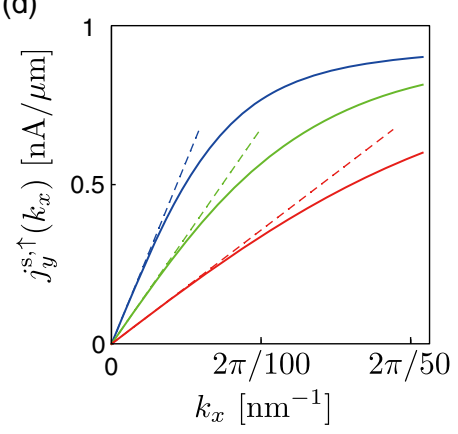

FIG. 3. (a) Weighted shift vector $\mathbf{R}^{\uparrow}$ (p) for the BHZ model at $T=0$ where the black contours denote EC contributing to the shift current dipole. The relative sizes and colors denote the relative magnitude of the vector field. Shift current dipole (picoamperes) (b) as a function of chemical potential $\mu$ at a fixed plasmon energy $\hbar \omega=200 \mathrm{meV}$ [38], and (c) as a function of plasmon energy $\hbar \omega$ with a fixed $\mu=-\hbar \omega / 2$ at different temperatures ( $T=10,20,40 \mathrm{~K}$ for blue, green, red curves). (d) Shift current calculated directly from Eq. (3) (solid curves) versus linear approximation from shift current dipole (dashed lines). Illustrative parameters used for the two-band model: $m_{0}=0.2 \mathrm{eV}, \quad c_{x}=6 \mathrm{eV} \AA^{2}, \quad c_{y}=3 \mathrm{eV} \AA^{2}, \quad v_{x}=1 \mathrm{eV} \AA$, $v_{y}=0.2 \mathrm{eV} \AA$, of a system with an inversion center and a mirror plane, e.g., a monolayer $1 \mathrm{~T}^{\prime}-\mathrm{MX}_{2}$ [44-46]. Here we used a plasmon electric field with $E=2000 \mathrm{~V} \mathrm{~cm}^{-1}$ [40].

$$
D_{a b}^{\uparrow}=\frac{C}{2} \int_{\mathbf{p}} \frac{\partial \epsilon_{v, \mathbf{p}}}{\partial p_{a}} R_{b}^{\uparrow}(\mathbf{p}) \delta\left(\omega_{\mathbf{p}}^{c v}-\omega\right) \delta\left(\epsilon_{v, \mathbf{p}}-\mu\right),
$$

where we have dropped mention of $\mathbf{k}=0$ in $\mathbf{R}$ for brevity. We plot $\mathbf{R}^{\uparrow}$ (p) in Fig. 3(a), which shows its pseudovector nature with respect to the mirror plane along the $x$ axis, Eq. (8). By integrating over the EC, we obtain a vanishing $D_{x x}^{\uparrow}$ and a nonzero $D_{x y}^{\uparrow}$. This gives a purely transverse $D_{a b}^{\uparrow}$ as expected from the above symmetry analysis.

We now turn to the finite temperature behavior of $D_{x y}^{\uparrow}$. In Fig. 3(b), we fix the polariton frequency (GP frequency) $\hbar \omega_{0}$ and plot $D_{x y}^{\uparrow}$ in Eq. (6) for $H_{0}$ in Eq. (10) for various chemical potentials and temperature values. Figure 3(b) displays a peaked $D_{x y}^{\uparrow}$ [with a width over a sizeable energy window: 10 to $20 \mathrm{meV}$ ] representing pronounced $D_{x y}^{\uparrow}$ when the Fermi surface crosses the EC; similarly, the width 
increases as temperature increases. Figure 3(b) also shows that peak $D_{x y}^{\uparrow}$ appears at $\mu=-\hbar \omega / 2$, i.e., when FS and EC overlap greatest with each other. When we fix $\mu=-\hbar \omega / 2$ and compute $D_{x y}^{\uparrow}$ as a function of $\hbar \omega$ in Fig. 3(c), we find $D_{x y}^{\uparrow}$ manifests over a wide window of photon and polariton energies.

In Fig. 3(d), we compare the up-spin photon-drag shift current $j_{y}^{s, \uparrow}\left(k_{x}\right)$ calculated from a direct integration from Eq. (3) and that from linear approximation in Eq. (5). For small $k_{x}, j_{y}^{\mathrm{s}, \uparrow}\left(k_{x}\right)$ grows linearly with $k_{x}$ as expected from Eq. (5) [dashed and solid lines coincide]. At low temperatures $T$, the transverse shift current begins to saturate at large $k_{x}\left(\lambda_{p}<100 \mathrm{~nm}\right)$; at higher $T$, the linear region becomes wider due to a temperature smeared out Fermi surface. Saturation arises when EC maximally tilts $|\mathbf{k}| \gtrsim|\mathbf{p}|$. As expected from the symmetry analysis above, the photon-drag shift current in Fig. 3(d) is purely transverse. We note that due to IS and TRS [e.g., found in Eq. (10)], $\mathbf{j}^{s, \uparrow}\left(k_{x}\right)=-\mathbf{j}^{s, \downarrow}\left(k_{x}\right)$ leading to spin photon-drag shift currents that propagate in opposite directions for spin $\uparrow, \downarrow$. Such spin currents (induced by linearly polarized light) propagate transversely to $\mathbf{k}$.

A finite linearly polarized charge photon-drag shift current, however, can be readily revealed when TRS is broken. One way to achieve this is via a Zeeman effect that splits the spin degeneracy: $H=H_{0}+H_{B} \quad$ with $H_{B}=\Delta s_{z} \tau_{0}$, which can be induced by a perpendicular magnetic field, or stacking another layer of magnetic material such as $\mathrm{CrI}_{3}$ on top of the target crystal [47-49]. Broken TRS imbalances $\mathbf{j}^{s, \uparrow}\left(k_{x}\right)$ and $\mathbf{j}^{s, \downarrow}\left(k_{x}\right)$ leading to a finite charge photon-drag shift current. Importantly, we emphasize that $H_{0}+H_{B}$ still preserves the composite symmetry $\mathcal{S}=\mathcal{M}_{y} \mathcal{T}$, see [36]. As a result, Eq. (8) still applies, guaranteeing a purely transverse $\mathbf{j}^{s}$.

Photon-drag shift current is a quantum geometric effect that proceeds directly from the subtle wave function coherences between conduction and valence bands as captured by $\mathbf{r}(\mathbf{p}, \mathbf{k})$. Indeed, $\mathbf{r}(\mathbf{p}, \mathbf{k})$ is the gradient of the Pacharatnam-Berry phase $[34,35]$ associated with the interband transition, see [36]. Arising even in centrosymmetric crystals, the photon-drag shift current's behavior can be described by a shift-current dipole $D_{a b}$ that quantifies the susceptibility of IS materials to interband (geometrical) effects; this parallels the Berry curvature dipole that captures the intraband nonlinear Hall effect in crystals with TRS. While here we have focused on linearly polarized photon-drag shift current, the same nonvertical transitions can similarly unblock circular shift current that are typically thought to vanish in a centrosymmetric system. Interestingly, circularly polarized photon-drag shift currents lead to a finite charge current that is controlled by the chirality of light, see discussion in [36]. Looking forward, we anticipate that other bulk photovoltaic currents (e.g., the injection current) and nonlinear responses can also be enriched by photon drag. Such nonvertical transition processes can be used to enlarge the set of materials where quantum geometric and Berry curvature related phenomena can be induced.

L.-K. S. gratefully acknowledges helpful conversations with Inti Sodemann. J. C. W. S. acknowledges support from the National Research Foundation (NRF), Singapore under its NRF fellowship program Award No. NRF-NRFF201605, the Ministry of Education, Singapore under its MOE AcRF Tier 3 Grant No. MOE2018-T3-1-002, and a Nanyang Technological University start-up grant (Grant No. NTU-SUG). K. C. is supported by the MOST of China No. 2017YFA0303400, the Chinese Academy of Sciences No. QYZDJSSW-SYS001, No. XDB28000000 and No. XDPB22.

* Corresponding author.

likunshi@pks.mpg.de

Corresponding author. justinsong@ntu.edu.sg

[1] A. G. Chynoweth, Surface space-charge layers in barium titanate, Phys. Rev. 102, 705 (1956).

[2] F. S. Chen, Optically induced change of refractive indices in $\mathrm{LiNbO}_{3}$ and $\mathrm{LiTaO}_{3}$, J. Appl. Phys. 40, 3389 (1969).

[3] A. M. Glass, D. von der Linde, and T. J. Negran, Highvoltage bulk photovoltaic effect and the photorefractive process in $\mathrm{LiNbO}_{3}$, Appl. Phys. Lett. 25, 233 (1974).

[4] V. I. Belinicher and B. I. Sturman, The photogalvanic effect in media lacking a center of symmetry, Sov. Phys. Usp. 23, 199 (1980).

[5] B. I. Sturman and V. M. Fridkin, The Photovoltaic and Photorefractive Effects in Noncentrosymmetric Materials (Gordon and Breach, New York, 1992).

[6] V. M. Fridkin, Bulk photovoltaic effect in noncentrosymmetric crystals, Crystallogr. Rep. (Transl. Kristallografiya) 46, 654 (2001).

[7] L. Z. Tan, F. Zheng, S. M. Young, F. Wang, S. Liu, and A. M. Rappe, Shift current bulk photovoltaic effect in polar materials - hybrid and oxide perovskites and beyond, npj Comput. Mater. 2, 16026 (2016).

[8] R. von Baltz and W. Kraut, Theory of the bulk photovoltaic effect in pure crystals, Phys. Rev. B 23, 5590 (1981).

[9] J. E. Sipe and A. I. Shkrebtii, Second-order optical response in semiconductors, Phys. Rev. B 61, 5337 (2000).

[10] S. M. Young and A. M. Rappe, First Principles Calculation of the Shift Current Photovoltaic Effect in Ferroelectrics, Phys. Rev. Lett. 109, 116601 (2012).

[11] L.Z. Tan and A. M. Rappe, Enhancement of the Bulk Photovoltaic Effect in Topological Insulators, Phys. Rev. Lett. 116, 237402 (2016).

[12] T. Rangel, B. M. Fregoso, B. S. Mendoza, T. Morimoto, J. E. Moore, and J. B. Neaton, Large Bulk Photovoltaic Effect and Spontaneous Polarization of Single-Layer Monochalcogenides, Phys. Rev. Lett. 119, 067402 (2017).

[13] M. Nakamura, S. Horiuchi, F. Kagawa, N. Ogawa, T. Kurumaji, Y. Tokura, and M. Kawasaki, Shift current photovoltaic effect in a ferroelectric charge-transfer complex, Nat. Commun. 8, 281 (2017). 
[14] A. M. Cook, B. M. Fregoso, F. de Juan, S. Coh, and J. E. Moore, Design principles for shift current photovoltaics, Nat. Commun. 8, 14176 (2017).

[15] M.-M. Yang, D. J. Kim, and M. Alexe, Flexo-photovoltaic effect, Science 360, 904 (2018).

[16] A. M. Burger, R. Agarwal, A. Aprelev, E. Schruba, A. Gutierrez-Perez, V. M. Fridkin, and J. E. Spanier, Direct observation of shift and ballistic photovoltaic currents, Sci. Adv. 5, eaau5588 (2019).

[17] J.Ahn, G.-Y. Guo, and N. Nagaosa, Low-Frequency Divergence and Quantum Geometry of the Bulk Photovoltaic Effect in Topological Semimetals, Phys. Rev. X 10, 041041 (2020).

[18] L. Gao, Z. Addison, E. J. Mele, and A. M. Rappe, Intrinsic fermi surface contribution to the circular photogalvanic effect, arXiv:2011.06542.

[19] T. Morimoto and N. Nagaosa, Topological nature of nonlinear optical effects in solids, Sci. Adv. 2, e1501524 (2016).

[20] H. Wang and X. Qian, Ferroicity-driven nonlinear photocurrent switching in time-reversal invariant ferroic materials, Sci. Adv. 5, eaav9743 (2019).

[21] Y. Kurman, N. Rivera, T. Christensen, S. Tsesses, M. Orenstein, M. Soljačić, J. D. Joannopoulos, and I. Kaminer, Control of semiconductor emitter frequency by increasing polariton momenta, Nat. Photonics 12, 423 (2018).

[22] I. Sodemann and L. Fu, Quantum Nonlinear Hall Effect Induced by Berry Curvature Dipole in Time-Reversal Invariant Materials, Phys. Rev. Lett. 115, 216806 (2015).

[23] G. Ribakovs and A. A. Gundjian, Theory of the photon drag effect in tellurium, J. Appl. Phys. 48, 4609 (1977).

[24] A. A. Grinberg and S. Luryi, Theory of the photon-drag effect in a two-dimensional electron gas, Phys. Rev. B 38, 87 (1988).

[25] E. L. Ivchenko and G. E. Pikus, New photogalvanic effect in gyrotropic crystals, JETP Lett. 27, 604 (1978).

[26] J. H. Strait, G. Holland, W. Zhu, C. Zhang, B. R. Ilic, A. Agrawal, D. Pacifici, and H.J. Lezec, Revisiting the Photon-Drag Effect in Metal Films, Phys. Rev. Lett. 123, 053903 (2019).

[27] E. L. Ivchenko, Circular photogalvanic effect in nanostrucutures, Phys. Usp. 45, 1299 (2002).

[28] S. D. Ganichev, E. L. Ivchenko, S. N. Danilov, J. Eroms, W. Wegscheider, D. Weiss, and W. Prettl, Conversion of Spin into Directed Electric Current in Quantum Wells, Phys. Rev. Lett. 86, 4358 (2001).

[29] T. Hatano, T. Ishihara, S. G. Tikhodeev, and N. A. Gippius, Transverse Photovoltage Induced by Circularly Polarized Light, Phys. Rev. Lett. 103, 103906 (2009).

[30] J. Karch et al., Dynamic Hall Effect Driven by Circularly Polarized Light in a Graphene Layer, Phys. Rev. Lett. 105, 227402 (2010).

[31] N. V. Proscia, M. Moocarme, R. Chang, I. Kretzschmar, V. M. Menon, and L. T. Vuong, Control of photo-induced voltages in plasmonic crystals via spin-orbit interactions, Opt. Express 24, 10402 (2016).

[32] M. Akbari, J. Gao, and X. Yang, Generation of transverse photo-induced voltage in plasmonic metasurfaces of triangle holes, Opt. Express 26, 21194 (2018).

[33] V. A. Shalygin, M. D. Moldavskaya, S. N. Danilov, I. I. Farbshtein, and L. E. Golub, Circular photon drag effect in bulk tellurium, Phys. Rev. B 93, 045207 (2016).

[34] N. A. Sinitsyn, Q. Niu, and A. H. MacDonald, Coordinate shift in the semiclassical Boltzmann equation and the anomalous Hall effect, Phys. Rev. B 73, 075318 (2006).

[35] L.-K. Shi and J. C. W. Song, Shift vector as the geometric origin of beam shifts, Phys. Rev. B 100, 201405(R) (2019).

[36] See the Supplemental Material at http://link.aps.org/ supplemental/10.1103/PhysRevLett.126.197402 for additional theoretical details.

[37] J. Ibañez-Azpiroz, I. Souza, and F. de Juan, Directional shift current in mirror-symmetric BC2N, Phys. Rev. Research 2, 013263 (2020).

[38] D. N. Basov, M. M. Fogler, and F. J. García de Abajo, Polaritons in van der Waals materials, Science 354, aag 1992 (2016).

[39] G. X. Ni et al., Fundamental limits to graphene plasmonics, Nature (London) 557, 530 (2018).

[40] F. R. Pratama, M. S. Ukhtary, and R. Saito, Non-vertical optical transition in near-field enhanced spectroscopy of graphene, J. Phys. Condens. Matter 31, 265701 (2019).

[41] J. N. Chen et al., Optical nano-imaging of gate-tunable graphene plasmons, Nature (London) 487, 77 (2012).

[42] Z. Fei et al., Gate-tuning of graphene plasmons revealed by infrared nano-imaging, Nature (London) 487, 82 (2012).

[43] B. A. Bernevig, T. L. Hughes, and S.-C. Zhang, Quantum spin Hall effect and topological phase transition in $\mathrm{HgTe}$ quantum wells, Science 314, 1757 (2006).

[44] X. Qian, J. Liu, L. Fu, and J. Li, Quantum spin Hall effect in two-dimensional transition metal dichalcogenides, Science 346, 1344 (2014).

[45] S.-Y. Xu et al., Electrically switchable Berry curvature dipole in the monolayer topological insulator $\mathrm{WTe}_{2}$, Nat. Phys. 14, 900 (2018).

[46] L.-K. Shi and J. C. W. Song, Symmetry, spin-texture, and tunable quantum geometry in a $\mathrm{WTe}_{2}$ monolayer, Phys. Rev. B 99, 035403 (2019).

[47] W. Zhao et al., Magnetic proximity and nonreciprocal current switching in a monolayer $\mathrm{WTe}_{2}$ helical edge, Nat. Mater. 19, 503 (2020).

[48] C.-X. Liu, X.-L. Qi, Xi Dai, Z. Fang, and S.-C. Zhang, Quantum Anomalous Hall Effect in $\mathrm{Hg}_{1-y} \mathrm{Mn}_{y}$ Te Quantum Wells, Phys. Rev. Lett. 101, 146802 (2008).

[49] C.-Z. Chang et al., Experimental observation of the quantum anomalous Hall effect in a magnetic topological insulator, Science 340, 167 (2013). 